Original paper

\title{
Evaluation of a breast software model for 2D and 3D X-ray imaging studies of the breast
}

\author{
Yanka Baneva ${ }^{\mathrm{a}}$, Kristina Bliznakova ${ }^{\mathrm{b}, *}$, Lesley Cockmartin ${ }^{\mathrm{c}}$, Stoyko Marinov ${ }^{\mathrm{b}}$, Ivan Buliev ${ }^{\mathrm{b}}$, \\ Giovanni Mettivier ${ }^{\mathrm{d}}$, Hilde Bosmans ${ }^{\mathrm{c}}$, Paolo Russo ${ }^{\mathrm{d}}$, Nicholas Marshall ${ }^{\mathrm{c}}$, Zhivko Bliznakov ${ }^{\mathrm{b}}$ \\ a Department of Physics and Biophysics, Medical University of Varna, Varna, Bulgaria \\ ${ }^{\mathrm{b}}$ Laboratory of Computer Simulations in Medicine, Technical University of Varna, Varna 9010, Bulgaria \\ ${ }^{\mathrm{c}}$ Medical Imaging Research Center, Department of Radiology, University Hospitals Leuven, Herestraat 49, 3000 Leuven, Belgium \\ ${ }^{\mathrm{d}}$ Università di Napoli Federico II, Dipartimento di Fisica "Ettore Pancini", and INFN Sezione di Napoli, Napoli, Italy
}

\section{A R T I C L E I N F O}

\section{Article history:}

Received 7 January 2017

Received in Revised form 31 March 2017

Accepted 22 April 2017

Available online 5 May 2017

\section{Keywords:}

Breast software model

Mammography

Breast software phantom

Breast imaging techniques

\begin{abstract}
A B S T R A C T
Introduction: In X-ray imaging, test objects reproducing breast anatomy characteristics are realized to optimize issues such as image processing or reconstruction, lesion detection performance, image quality and radiation induced detriment. Recently, a physical phantom with a structured background has been introduced for both 2D mammography and breast tomosynthesis. A software version of this phantom and a few related versions are now available and a comparison between these 3D software phantoms and the physical phantom will be presented.

Methods: The software breast phantom simulates a semi-cylindrical container filled with spherical beads of different diameters. Four computational breast phantoms were generated with a dedicated software application and for two of these, physical phantoms are also available and they are used for the side by side comparison. Planar projections in mammography and tomosynthesis were simulated under identical incident air kerma conditions. Tomosynthesis slices were reconstructed with an in-house developed reconstruction software. In addition to a visual comparison, parameters like fractal dimension, power law exponent $\beta$ and second order statistics (skewness, kurtosis) of planar projections and tomosynthesis reconstructed images were compared.

Results: Visually, an excellent agreement between simulated and real planar and tomosynthesis images is observed. The comparison shows also an overall very good agreement between parameters evaluated from simulated and experimental images.

Conclusion: The computational breast phantoms showed a close match with their physical versions. The detailed mathematical analysis of the images confirms the agreement between real and simulated 2D mammography and tomosynthesis images. The software phantom is ready for optimization purpose and extrapolation of the phantom to other breast imaging techniques.
\end{abstract}

(C) 2017 Associazione Italiana di Fisica Medica. Published by Elsevier Ltd. This is an open access article under the CC BY-NC-ND license (http://creativecommons.org/licenses/by-nc-nd/4.0/).

\section{Introduction}

Physical and computational phantoms are essential for the development, optimization, and evaluation of modern X-ray imaging systems. Modern breast imaging techniques can be two-dimensional (digital mammography, dual energy and contrast-enhanced mammography, the advanced monochromatic and phase-contrast imaging [1-4]) or three-dimensional (tomosynthesis and breast CT [513]). For all these techniques, it is important that both physical and software phantoms reproduce the breast characteristics for the study of particular aspects of the imaging chain such as image

\footnotetext{
* Corresponding author.

E-mail address: kristina.bliznakova@tu-varna.bg (K. Bliznakova).
}

processing or reconstruction, lesion detection performance, image quality and radiation induced detriment. In most cases, physical breast phantoms are manufactured from a homogeneous mixture and this may result in a lack of realistic 2D and 3D image representation. While many groups work at a 3D anthropomorphic phantom [14-28], there is not yet a consensus on a particular multi-purpose phantom. The degree of realism of the glandular structure is also variable and inserting lesion like objects remains a challenge. On top of this, physical phantoms may be expensive, especially if different breast models have to be foreseen.

These are some of the reasons that lead to the introduction of computational phantoms and their use in virtual experiments in X-ray imaging. The best computational phantoms allow detailed modelling of the 3D structures of the breast and produce image 
characteristics similar to these produced by radiation of real breasts. Assessment of agreement between simulated and real images has been done with fractal dimension and the power law exponent $\beta$ of the logarithmic power spectrum of mammographic projection images, as well as other parameters such as first order, second order or higher order statistical parameters.

One worked-out example is the UPenn breast phantom that was designed as a software phantom and later realized for physical measurements of 2D mammography and breast tomosynthesis [14-16,21]. Another physical phantom has been introduced by Cockmartin et al. [29] which produces a structured background and was tested for both 2D mammography and breast tomosynthesis [30-32]. This physical breast phantom is based on the work of Gang et al. [33], where it is shown that equal volumes of differently sized acrylic spheres provide a fractal dimension of 3 as well as a power law exponent $\beta$ equal to 3 . The phantom consists of acrylic spheres placed in an acrylic container filled either with air or with water. The phantom images showed power law exponents in the range of the exponents measured in patient data [31].

Further development of this phantom are expected based on the improvement of the inserted breast lesions, the improvement of the shape of the beads (non-spherical) and the study of more appropriate material compositions (other than PMMA and water) for the manufacturing of the phantom. For this purpose, a software application called LUCMFRGen was developed that allows to create software versions of the physical phantom based on parameters like sizes of the container and the spheres, as well as the material characteristics. In the beginning, the tool was developed for educational applications [34].

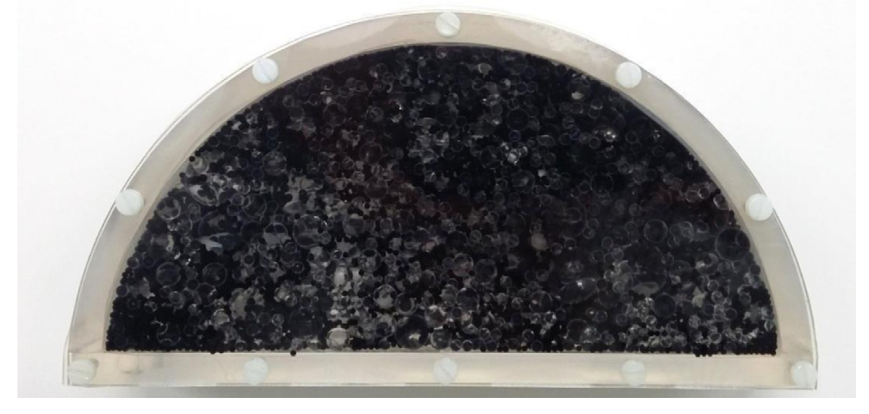

Fig. 1. A photo of the physical breast phantom, filled with water
The purpose of this work was to perform a detailed validation of the LUCMFRGen software for applications in mammography and tomosynthesis.

\section{Materials and methods}

\subsection{Physical phantom}

The physical phantom is an acrylic semi-circular container with $48 \mathrm{~mm}$ thickness and $200 \mathrm{~mm}$ diameter (Fig. 1), representing $60 \mathrm{~mm}$ compressed breast thickness [32]. Acrylic spheres with six different diameters were inserted in the container: $15.88 \mathrm{~mm}$, $12.70 \mathrm{~mm}, 9.52 \mathrm{~mm}, 6.35 \mathrm{~mm}, 3.18 \mathrm{~mm}$ and $1.85 \mathrm{~mm}$. Compared to Gang's publication [33], spheres with smaller diameters have been added. The total volume of the acrylic spheres of one particular diameter was constant [29]. This phantom is dedicated to testing and optimizing 2D mammography and breast tomosynthesis.

Two such physical phantoms were initially produced: one phantom of acrylic spheres filled with air and one phantom filled with water (Fig. 1).

\subsection{Software phantom}

In order to create phantoms with different dimensions, volume and content, a dedicated software tool LUCMFRGen was designed, implemented and used in this study [34]. The computational model was divided in two main parts - a container and a spheres. The container is modelled as a set of two semi-cylinders and a slab object, approximating the sidewall of the physical model. The software application samples randomly the location of the spherical objects within the internal semi-cylinder, starting from the spheres with largest diameter. The algorithm also verifies that the currently sampled sphere does not have intersection with other spheres. The procedure of generating spheres is time consuming, as their number reaches 29,000 and every new sphere needs to be checked for non-overlapping. The algorithm implements the sphere packing concept for unequal non-overlapping spheres in a container space. Since we use random packing of the generated spheres, the packing density of the spheres is approximately $64 \%$. The software was tested for educational applications in an international course program (Eutempe-Rxmodule 5) [34,35].

For the purposes of this work, four phantoms which have equal container dimensions were created. An example is shown in Fig. 2b and summarized in Table 1.

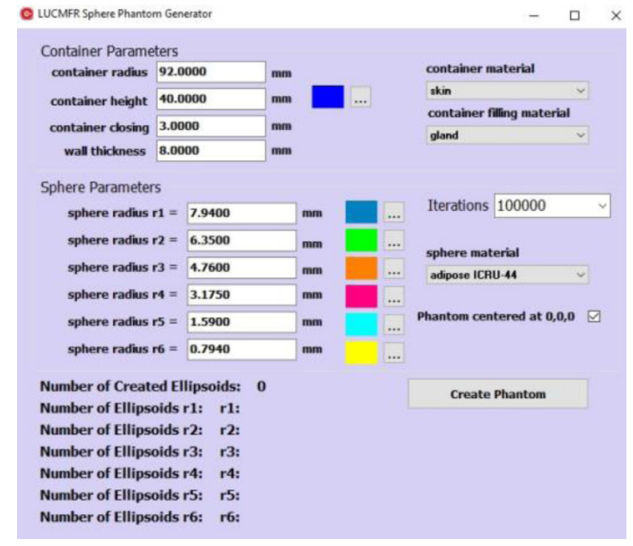

(a)

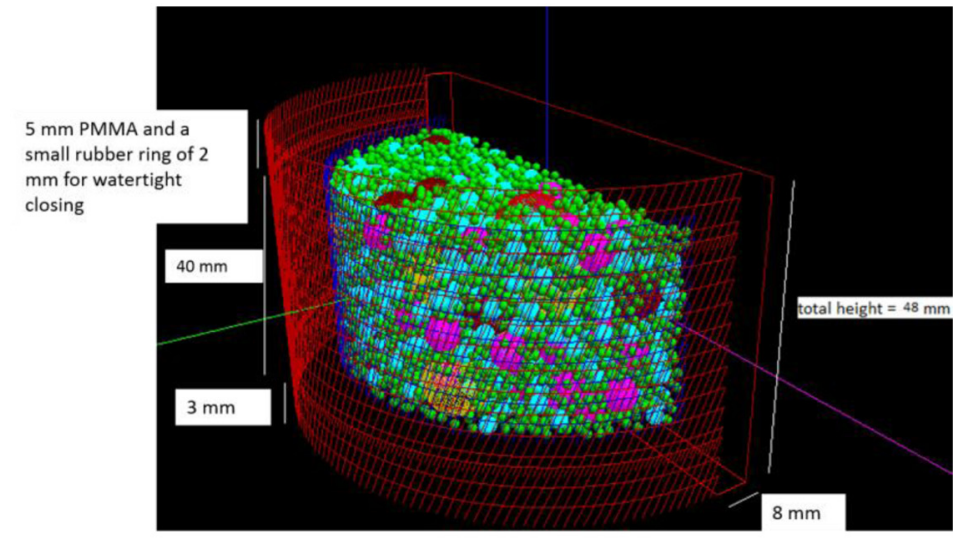

(b)

Fig. 2. Generation of computational breast phantoms: (a) a screenshot from the LUCMFRGen software and (b) generated phantom. 
Table 1

Description of the computational breast phantoms, generated with the LUCMFRGen software tool.

\begin{tabular}{llll}
\hline Phantoms & Container & Filling material & Spheres \\
\hline Phantom1 & PMMA & Air & PMMA \\
Phantom2 & PMMA & Water & PMMA \\
Phantom3 & Skin & Glandular tissue & Adipose \\
Phantom4 & PMMA & Water & Polyethylene \\
\hline
\end{tabular}

The phantoms differ in the material composition, used for modelling the parts of the phantom: polymethyl methacrylate (PMMA), polyethylene, water, glandular and adipose tissue, skin and air. The composition of the elements of the materials is summarized in Table 2 and was taken from National Institute of Standard and Technology (NIST) database [36].

The first two phantoms reproduce already workable phantoms and can be used for validation purposes. Phantoms 3 and 4 illustrate potential developments, in particular phantom 3 aims to simulate a realistic presentation of the different breast tissues, namely a breast structure of adipose spherical compartments aligned by glandular strands. Moreover within a real breast more adipose tissue is present compared to glandular tissue. In this phantom the packing density is $64 \%$ resulting in $64 \%$ adipose tissue represented by the spheres and $36 \%$ glandular tissue as filling material. Phantom 4 represents a theoretical solution that could not be realized in practice due to miniscule holes in the polyethylene, that could not be avoided during production of the spheres. Results of phantoms 3 and 4 could be compared to phantoms 1 and 2 and subsequently to patient data.

\subsection{Acquisition of images from physical phantoms}

The Siemens Mammomat Inspiration system (Erlangen, Germany) was used to produce projections with $2800 \times 3518$ pixels and pixel pitch of $85 \mu \mathrm{m}$. The distances from the source to the breast support table and from the source to the detector are $633 \mathrm{~mm}$ and $650 \mathrm{~mm}$, respectively. Images of the physical phantoms 1 and 2 were acquired both in 2D and tomosynthesis mode. Breast tomosynthesis was performed with 25 projections images [9-10] and a total incident air kerma of $7.4 \mathrm{mGy}$.

\subsection{II.4. Simulation of 2D mammography and tomosynthesis}

Planar X-ray projection images were obtained for an X-ray beam energy of $28 \mathrm{kVp}$ and $\mathrm{W} / \mathrm{Rh}$ as anode filter combination (Fig. 3b) with the in-house developed XRAYImagingSimulator [37]. This software tool consists of two main modules: (a) a module for phantom modelling, based on solid and voxel geometry, and (b) a module for acquisition geometry and X-ray image formation modelling, and allows simulation of 2D and 3D X-ray imaging techniques such as radiography, tomosynthesis, cone beam CT.

In the present study, the phantom is built from solid primitives (Fig. 2b). The module for acquisition geometry is used to model 2D and 3D mammography. Common parameters of the simulated geometry include distances from the source to the center of rotation and to the detector (Fig. 3a), gantry acquisition arc and angular step, as well as imaging and beam parameters. For simulation purposes, distances from the source to the isocenter and to the detector surface were $603 \mathrm{~mm}$ and $650 \mathrm{~mm}$, respectively; X-ray projection images have $3500 \times 3500$ pixels and a pixel pitch of $85 \mu \mathrm{m}$. Fig. 4a shows a screenshot from the module used to acquire images of the X-ray imaging simulator [37]. X-ray projection images are obtained with simulation of X-ray photon transport through the phantom, based on the Beer-Lambert law. Scatter radiation at this stage was not considered. The obtained scatterfree images were appropriately modified to include photon noise. For tomosynthesis simulations, the incident air kerma at the surface of the breast phantom was set to $7.4 \mathrm{mGy}$, while for planar mammography imaging the incident air kerma was set to $4 \mathrm{mGy}$. The photon fluence was calculated as:

Table 2

Elemental composition as percentage weights and density of used breast substitutes in the study.

\begin{tabular}{|c|c|c|c|c|c|c|c|c|c|}
\hline & $H$ & C & $N$ & $O$ & $\mathrm{Na}$ & $P$ & $S$ & $\mathrm{Cl}$ & $\begin{array}{l}\text { Density } \\
\mathrm{g} / \mathrm{cm}^{3}\end{array}$ \\
\hline Adipose tissue & 0.114 & 0.598 & 0.007 & 0.278 & 0.001 & & 0.001 & 0.001 & 0.95 \\
\hline Gland & 0.106 & 0.332 & 0.030 & 0.527 & 0.001 & 0.001 & 0.002 & 0.001 & 1.02 \\
\hline PMMA & 0.080 & 0.600 & & 0.320 & & & & & 1.19 \\
\hline Polyethylene & 0.144 & 0.856 & & & & & & & 0.93 \\
\hline Liquid water & 0.120 & & & 0.880 & & & & & 1.00 \\
\hline
\end{tabular}

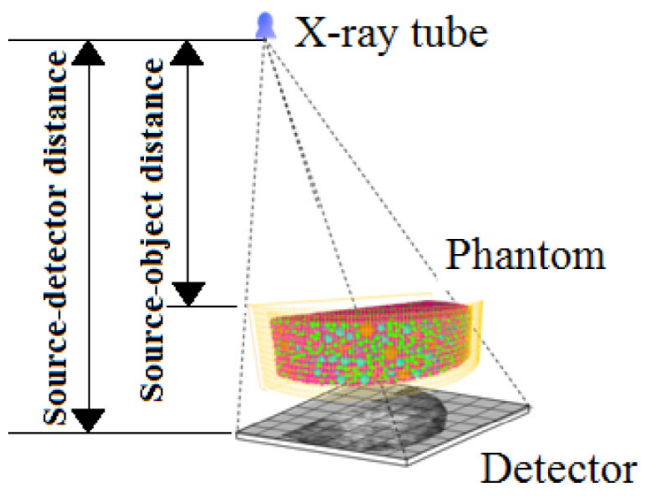

a)

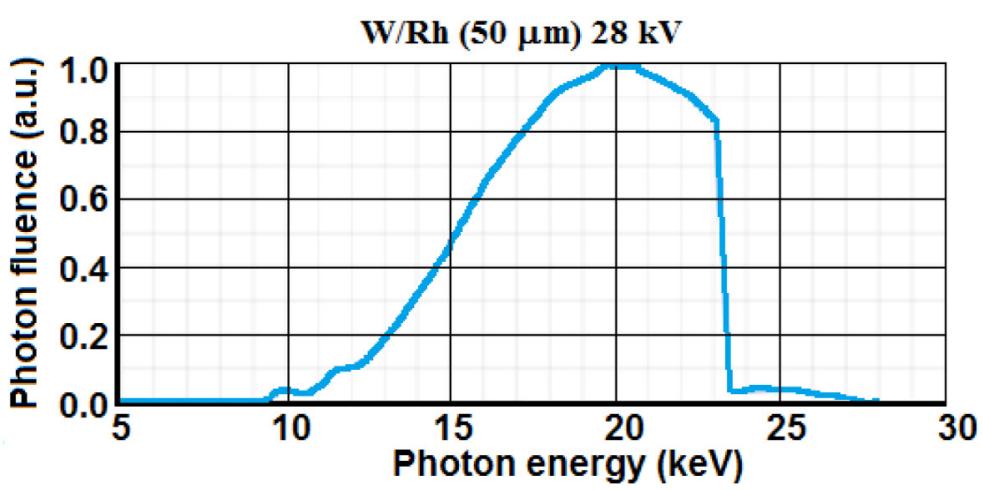

b)

Fig. 3. Image acquisition step: (a) simulated setup and (b) incident spectra beam. 


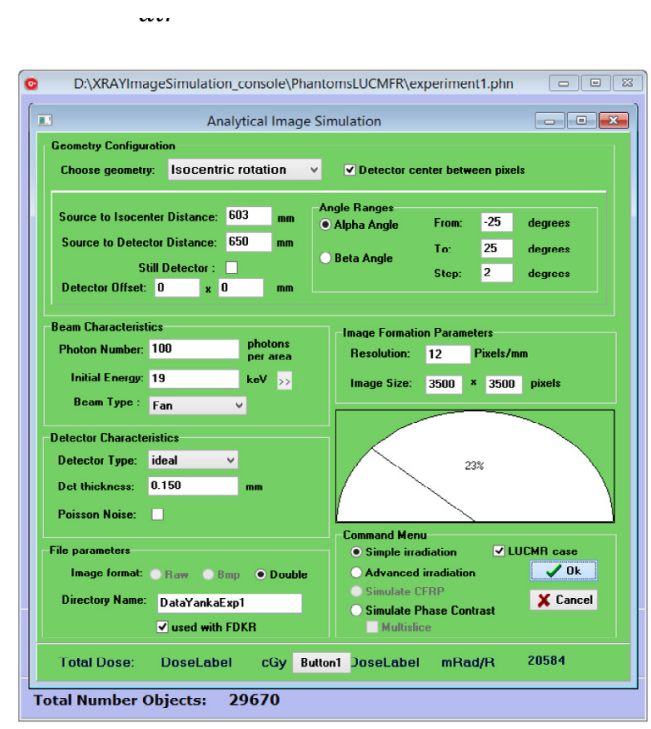

(a)

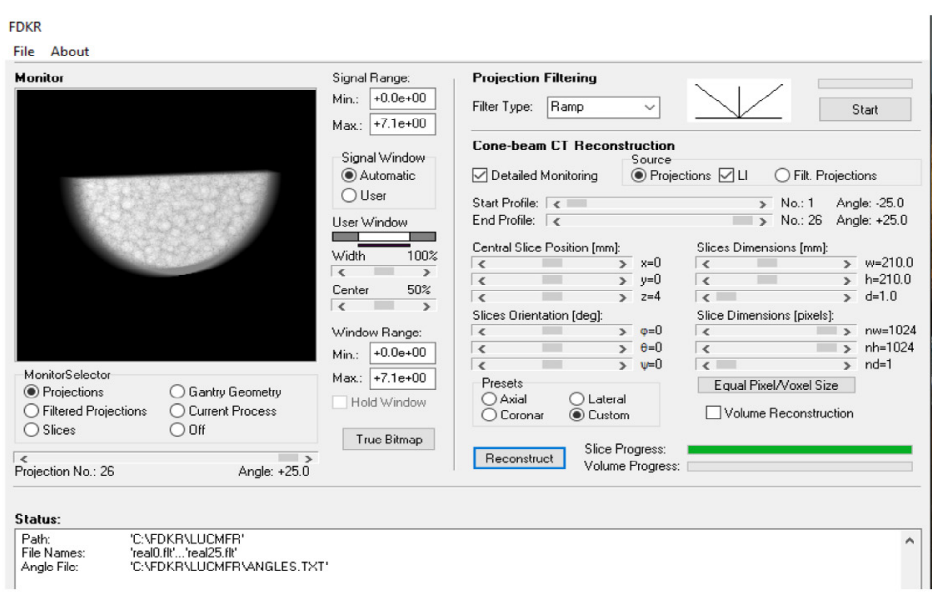

(b)

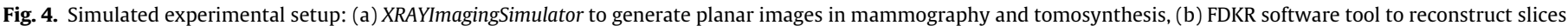
and the breast volume.

$K=\left[\frac{\mu_{e n}}{\rho}\right]_{\text {air }} \cdot E \cdot \Phi$

where $\mathrm{K}$ is the incident air kerma, $\mathrm{E}$ is the energy of the incident photons, $\Phi$ is the photon fluence and $\left[\frac{\mu_{e n}}{\rho}\right]_{a i r}$ is the mass energy absorption coefficients for air.

\subsection{Reconstruction of images}

Tomosynthesis slices were reconstructed with the in-house developed image reconstruction tool FDKR [38]. This tool allows quick browsing of projection images of different origin, as well as through stacks of reconstructed images of CBCT, CT and tomosynthesis (Fig. 4b). The image reconstruction is based on the FDK algorithm (circular scanning trajectory), which assumes onedimensional filtering of the projections using a high-pass filter with linearly increasing frequency response - the Ramp filter [39]. The tool allows using or not of two types of filters: pure high-pass Ramp filter and a customized, smoother version of the Ramp filter. The later results in reduction of the random noise in the reconstructed tomograms. FDKR has been built using objectoriented programming approach.

For this study, three planes of interest, including the central slice were reconstructed with a pixel size of $85 \mu \mathrm{m} \times 85 \mu \mathrm{m}$. Slice thickness was set $1 \mathrm{~mm}$. Images were reconstructed with both filtered backprojection algorithm.

\subsection{Evaluation of images}

Images were evaluated by using an in-house developed tool, dedicated to extract image features [40]. A screenshot of the platform is shown in Fig. 5. The software tool was created with the help of the GUIDE module in MATLAB (https://www.mathworks.com/products/matlab.html), which allows building of user interfaces as Windows applications. The platform's main window consists of three main parts for image load, region definition and feature calculation.

The application is adapted to work with both DICOM and non-formatted (raw) images. The latter are usually generated with simulation tools. Initially, the region of the breast phantom is segmented to obtain the contours of the object. Further, for the calculation of the skewness and kurtosis, the outlined area was divided into square regions with size of $50 \times 50$ pixels, while for the calculation of the fractal dimension and the $\beta$ parameter, $200 \times 200$ pixels. The sizes of the regions were selected according to [41]. For any selected region of interest, the software calculates first, second and higher order statistical data: standard deviation, skewness and kurtosis as described in Table 3. The average value for the mentioned features is calculated and presented together with the standard deviation. All these features are used in advanced CAD software applications to classify the breast as dense, fatty and glandular tissue ratios, as well as to identify the risk of breast cancer [41].

\section{Results and discussion}

Validation includes visual and quantitative comparison of 2D and tomosynthesis real and simulated images for phantom configurations 1 and 2. For comparison, planar and reconstructed images of the other two virtual phantom configurations are also shown and quantitatively evaluated.

\subsection{Validation for projection mammography}

Comparisons between simulated and experimental planar images of phantom configuration 1 (PMMA beads and air) and 2 (PMMA beads and water) are shown in Fig. 6.

The quantitative evaluation of simulated and experimental images is summarized in Table 4.

The images in Fig. 6a, $c$ are generated from phantoms filled with air, while images in Fig. 6 b, $d$ are related to the phantoms with filling material - water. A very good conformity between simulated and experimentally acquired images is observed visually for both phantom configurations. This subjective conclusion is well supported by the very good agreement between features extracted from experimental and simulated images. Fig. 6e, f show simulated planar images from phantoms 3 and 4, which present good visual appearance that is close to one produced from phantom 2 (with 


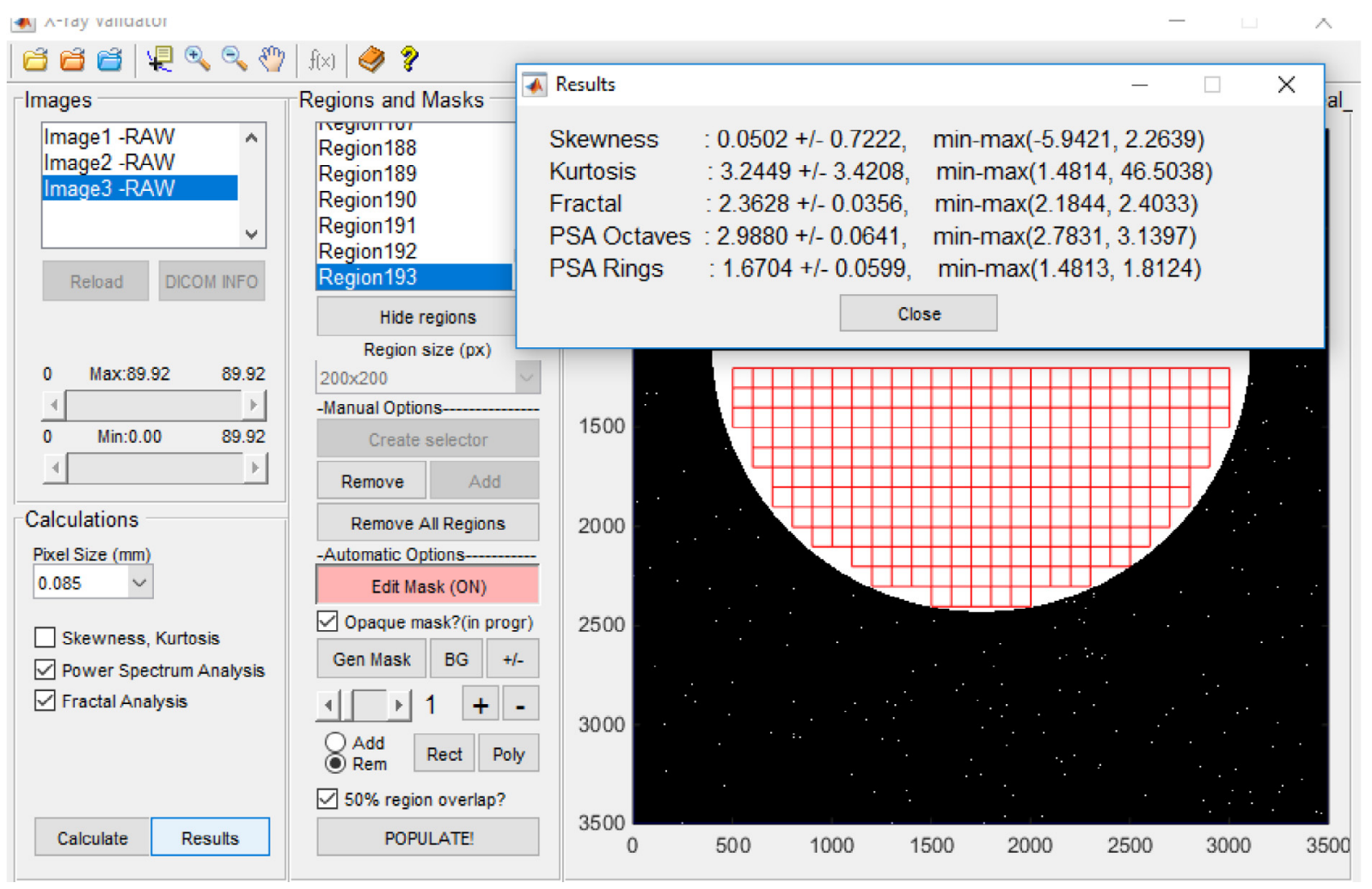

Fig. 5. A screenshot from the graphical user interface of the software used to evaluate features of interest from X-ray images.

Table 3

Features extracted from projection images and reconstructed tomograms.

\begin{tabular}{|c|c|c|c|}
\hline $\begin{array}{l}\text { Extracted } \\
\text { feature }\end{array}$ & Purpose & Formula & \\
\hline Kurtosis & $\begin{array}{l}\text { Measures the relative tailedness (relatively to the normal distribution) } \\
\text { or flatness of a distribution }\end{array}$ & $\begin{array}{l}\operatorname{Kurt}[\mathrm{X}]=\frac{\mu_{4}}{\sigma^{4}}=\frac{E\left[(X-\mu)^{4}\right]}{\left(E\left[(X-\mu)^{2}\right]\right)^{2}} \\
E \text { is the expectation operator, where } \mu_{4} \text { is the fourth moment about the } \\
\text { mean }(\mu) \text { and } \sigma \text { is the standard deviation }\end{array}$ & [2] \\
\hline Skewness & $\begin{array}{l}\text { Measures the asymmetry of the probability distribution of a real-valued } \\
\text { random variable about its mean. The skewness value can be positive or } \\
\text { negative, or even undefined. }\end{array}$ & $\begin{array}{l}\gamma_{1}=E\left[\left(\frac{X-\mu}{\sigma}\right)^{3}\right]=\frac{\mu_{3}}{\sigma^{3}}=\frac{E\left[(X-\mu)^{3}\right]}{\left(E\left[(X-\mu)^{2}\right]\right)^{\frac{3}{2}}}=\frac{\kappa_{3}}{\kappa_{2}^{3 / 2}} \\
\text { where } E \text { is the expectation operator, } \mu_{3} \text { is the third central moment }\end{array}$ & [3] \\
\hline $\begin{array}{l}\text { Fractal } \\
\quad \text { analysis }\end{array}$ & $\begin{array}{l}\text { Measures the fractal characteristics of the images. A lower fractal } \\
\text { dimension corresponds to smoother texture and opposite. }\end{array}$ & $\begin{array}{l}A(\varepsilon)=\sum_{\mathrm{x}, \mathrm{y}} \varepsilon^{2}+\sum_{\mathrm{x}, \mathrm{y}} \varepsilon\left(\left|\mathrm{i}_{\varepsilon}(x, y)-\mathrm{i}_{\varepsilon}(x, y+1)\right|+\left|\mathrm{i}_{\varepsilon}(x, y)-\mathrm{i}_{\varepsilon}(x+1, y)\right|\right) \\
\text { where } \mathrm{A}(\varepsilon) \text { is the area of the surface for a given pixel size } \varepsilon, \varepsilon^{2} \text { is sum of } \\
\text { the area of each pixel }\end{array}$ & [4] \\
\hline \multirow[t]{2}{*}{$\begin{array}{r}\text { Power law } \\
\text { spectral } \\
\text { analysis }\end{array}$} & $\begin{array}{l}\text { The power spectrum describes the signal and noise into frequency } \\
\text { components of which the low frequency range can be characterized by a } \\
\text { power law. The exponent of this power law gives then a measure of the } \\
\text { texture or anatomical clutter }\end{array}$ & $\begin{array}{l}F(k, l)=\frac{1}{\mathrm{MN}} \sum_{\mathrm{a}=0}^{\mathrm{M}-1} \sum_{\mathrm{b}=0}^{\mathrm{N}-1} \mathrm{f}(\mathrm{a}, \mathrm{b}) \exp \left[-2 \pi \mathrm{i}\left(\frac{\mathrm{ka}}{\mathrm{M}}+\frac{\mathrm{lb}}{\mathrm{N}}\right)\right] \\
\text { where } \mathrm{k}(0,1, \ldots, \mathrm{M}-1) \text { and } \mathrm{l}(0,1, \ldots \mathrm{N}-1) \text { are the relative spatial } \\
\text { frequencies in the two directions and } \mathrm{M} \times \mathrm{N} \text { is the image size of } \mathrm{f}(\mathrm{a}, \mathrm{b})\end{array}$ & [5] \\
\hline & & $\begin{array}{l}\text { The total power spectrum per concentric ring is further transformed to } \\
\log _{2} \text { (total power spectrum) and plotted versus the octave number [42]. } \\
\text { The plot is almost linear and the data are fitted to a line obtained using } \\
\text { linear regression analysis. The slope of the line } m \text { is related to } \\
\text { exponential parameter } \beta \text { as: } m=\beta-2\end{array}$ & \\
\hline
\end{tabular}

the water filling material). Comparison of calculated parameters from images taken from phantom 3 and phantom 4 shows that the phantom with the characteristics close to the real breast (phantom 3) presents values close to phantom 2 (PMMA-water). Also, the quantitative evaluation of phantom 4 shows that a phantom with such a design container based on PMMA, spheres made from polyethylene and filling material - water, is also a good candidate for such a physical breast phantom. The higher values of the error for kurtosis are due to the difference in the texture included in the regions which are used for the calculation.

\subsection{Validation for breast tomosynthesis}

Similarly to the 2D mammography, comparison between tomosynthesis images obtained from simulated and experimental projection images are shown in Fig. 7.

The side by side comparison of tomosynthesis images for phantom 1 (PMMA beads in air, Fig. 7a, c) and phantom 2 (PMMA beads in water, Fig. 7b, d) shows an excellent visual agreement between simulated and experimental tomograms. The observed blurring in the images is expected and it is mainly due to (1) the limited scan- 

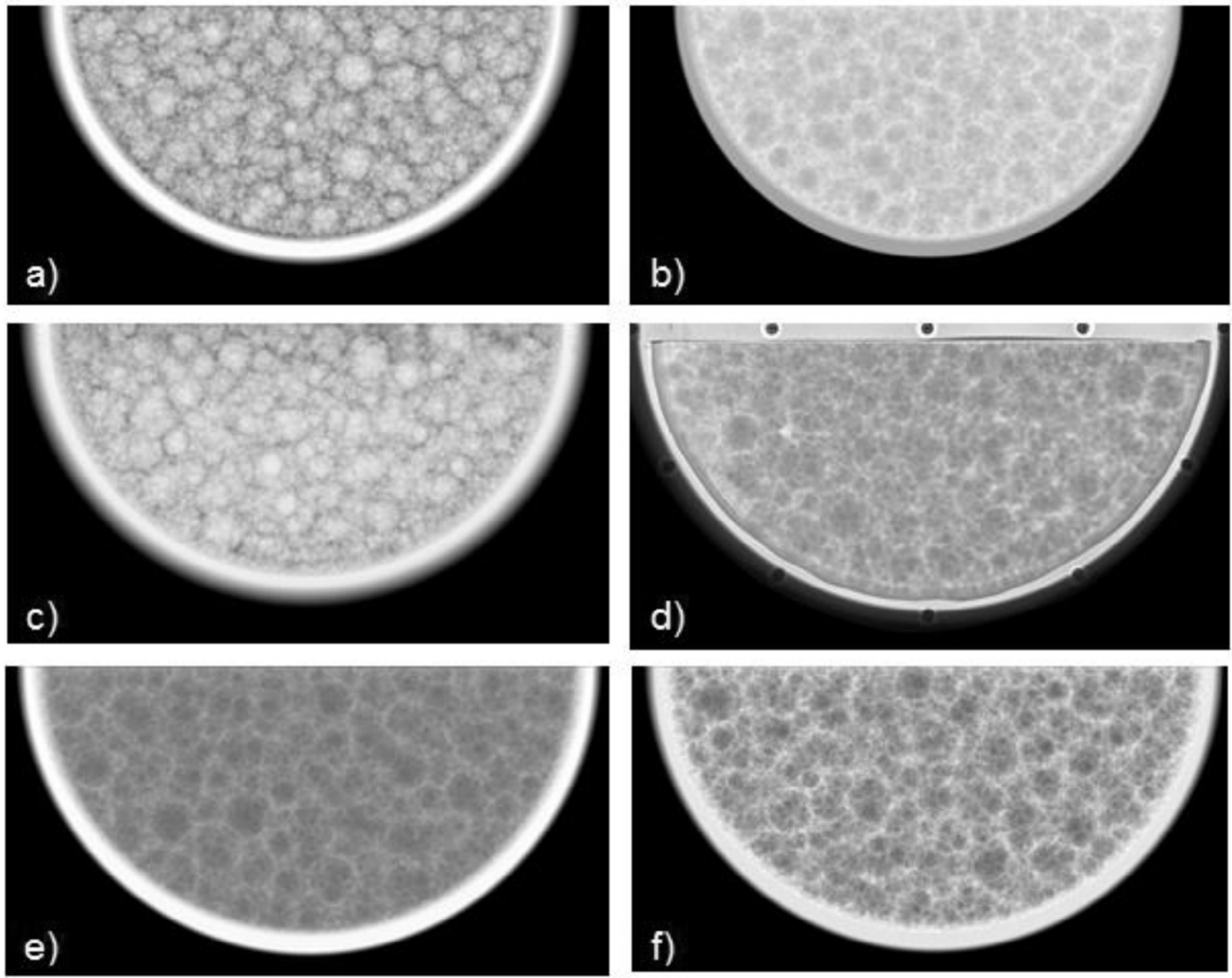

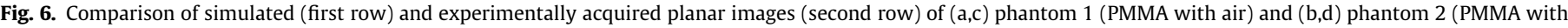

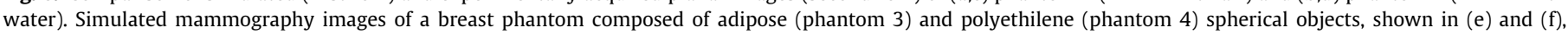
respectively.

Table 4

Quantitative evaluation of simulated and experimental mammography images.

\begin{tabular}{|c|c|c|c|c|}
\hline Phantom & Skewness & Kurtosis & Power law exponent $(\beta)$ & Fractal dimension \\
\hline Phantom 1 (real) & $0.72 \pm 0.40$ & $3.50 \pm 1.26$ & $3.23 \pm 0.07$ & $2.44 \pm 0.02$ \\
\hline Phantom 1 (sim) & $0.04 \pm 0.76$ & $3.34 \pm 3.99$ & $3.38 \pm 0.11$ & $2.40 \pm 0.01$ \\
\hline Phantom 2 (real) & $0.35 \pm 0.54$ & $3.13 \pm 1.62$ & $2.71 \pm 0.15$ & $2.08 \pm 0.07$ \\
\hline Phantom 2 (sim) & $0.09 \pm 0.71$ & $3.26 \pm 3.35$ & $2.63 \pm 0.11$ & $2.10 \pm 0.01$ \\
\hline Phantom 3 (sim) & $-0.30 \pm 0.68$ & $3.40 \pm 3.56$ & $2.40 \pm 0.17$ & $2.12 \pm 0.01$ \\
\hline Phantom 4 (sim) & $-0.002 \pm 0.93$ & $3.65 \pm 5.24$ & $3.27 \pm 0.01$ & $2.08 \pm 0.01$ \\
\hline
\end{tabular}

ning arc in the projection acquisition for the tomosynthesis, and (2) the conical profile of the X-ray beam (the filtered back-projection algorithm assumes that the detector rows acquire images from $\mathrm{X}$-rays lying in a plane but away from the source trajectory plane, that is not valid anymore). The visual comparison identifies similar appearance of simulated structures in the computational and physical phantoms. Logically, experimentally obtained tomograms appear more blurred compared to simulated. This is due to the fact that X-rays were not simulated within the detector.

Quantitatively, the evaluated features of the tomosynthesis images are shown in Table 5.

Comparison of $\beta$ between 2D and tomosynthesis slices shows lower $\beta$ values for tomosynthesis images. $\beta$ is lower in 3D reconstructed images compared to $2 \mathrm{D}$ projection images because of the reduced texture in the slices compared to the projections. images. This is also reported from other publications which concern real and simulated data [30,31]. For 2D mammography, there is an excellent coincidence between simulated and experimentally derived fractal dimensions. This is due to the large photon fluence used to form one planar mammography. On the contrary, due to the 25 images acquired during tomosynthesis with a total incident exposure equal to twice that used for the 2D images, the photon fluence per projection image is decreased, resulting in more noise and therefore higher fractal dimension. Beside this, there are also other sources of noise in the detector (not presently simulated), which also lead to higher values for the fractal dimension. The almost ideal properties of our detector resulted in less noise and therefore lower values for the fractal dimension 

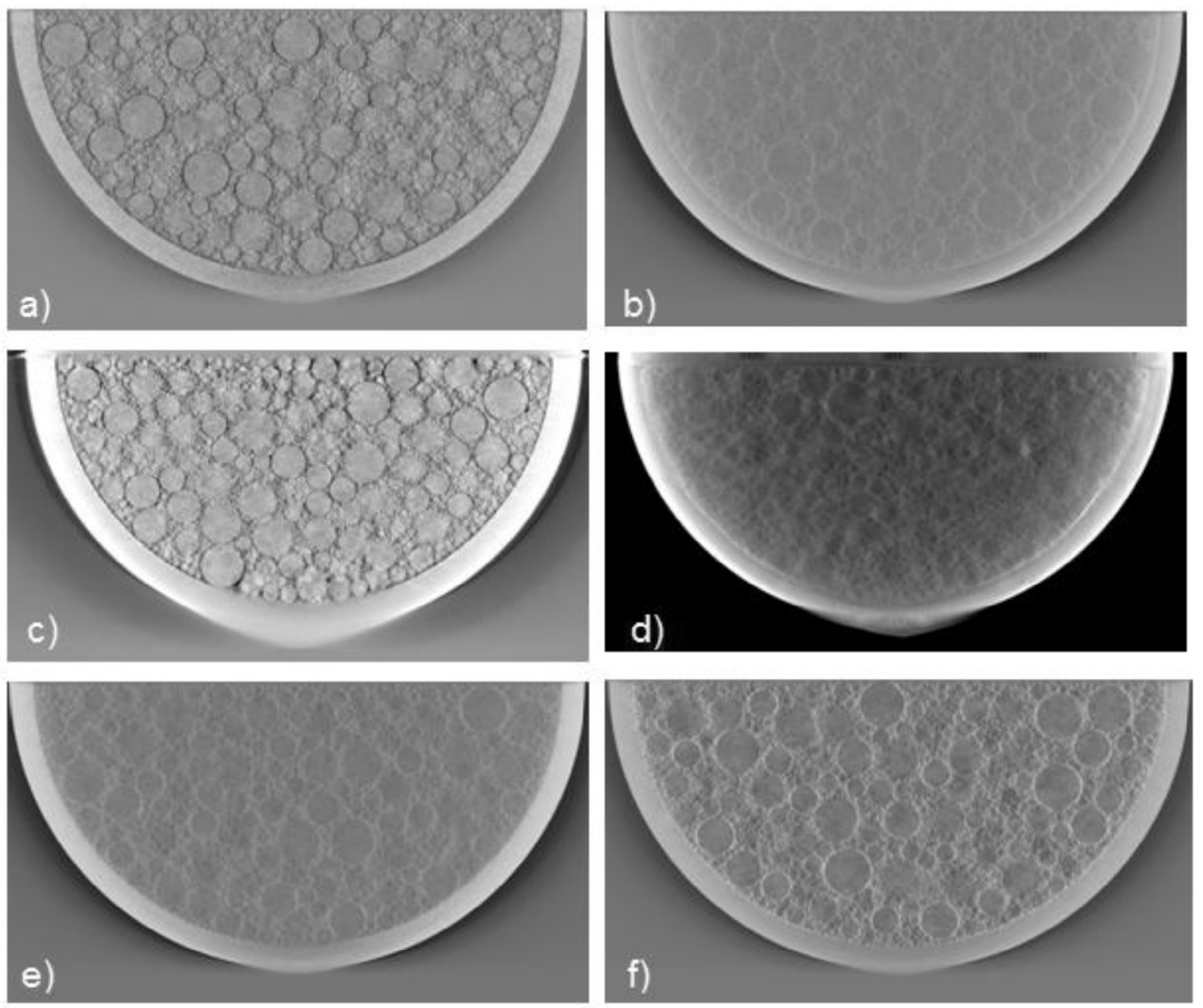

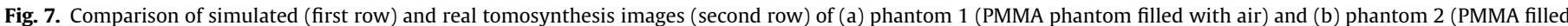

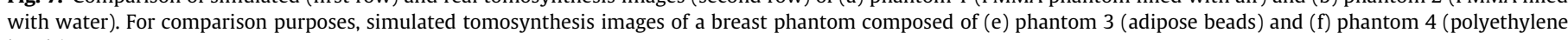
beads).

Table 5

Quantitative evaluation of tomosynthesis reconstruction simulated and experimental images.

\begin{tabular}{|c|c|c|c|c|}
\hline Phantom & Skewness & Kurtosis & Power law exponent $(\beta)$ & Fractal dimension \\
\hline Phantom 1 (real) & $-0.70 \pm 0.60$ & $3.88 \pm 2.09$ & $3.04 \pm 0.11$ & $2.57 \pm 0.04$ \\
\hline Phantom 1 (sim) & $-0.30 \pm 0.51$ & $3.95 \pm 0.57$ & $2.81 \pm 0.06$ & $2.21 \pm 0.01$ \\
\hline Phantom 2 (real) & $0.07 \pm 0.47$ & $3.35 \pm 2.04$ & $1.90 \pm 0.19$ & $3.21 \pm 0.05$ \\
\hline Phantom 2 (sim) & $-0.02 \pm 0.38$ & $3.14 \pm 2.06$ & $1.35 \pm 0.19$ & $2.56 \pm 0.02$ \\
\hline Phantom 3 (sim) & $1.00 \pm 0.48$ & $3.89 \pm 0.97$ & $1.59 \pm 0.03$ & $2.51 \pm 0.01$ \\
\hline Phantom 4 (sim) & $1.64 \pm 0.95$ & $6.66 \pm 4.11$ & $2.01 \pm 0.05$ & $2.47 \pm 0.01$ \\
\hline
\end{tabular}

for the simulated phantoms compared to the real. Finally, skewness and kurtosis were similar for both 2D and tomosynthesis images.

The results of this study were compared to the results demonstrated by the UPenn software breast phantom [43]. The $\beta$ values for phantom 2 , which closely resembles the properties of the real mammography texture are found to be similar to these reported by Bakic et al. [43] for the evaluation of the UPenn software phantom. The authors report an average $\beta$ value of 2.65 (the author calculated $2 \beta$, which corresponds to $\beta=1.325$ ) for $450 \mathrm{ml}$ phantoms and 2.62 (corresponding to 1.31 for our case) for $1500 \mathrm{ml}$ phantoms. Similarly, to this study, the authors did not include any quantum or detector noise and have not included scatter or detector blur.

In case of 3D imaging, lower values for $\beta$ are computed for simulated tomograms compared to real ones. This may be attributed to the fact that X-rays were not simulated. This is part of feature studies.

The evaluation suggests that both, the software phantom and the acquisition geometry are modelled in sufficient details in order to receive similar visual appearance. Efforts are focused on the simulation of the X-ray imaging by using Monte Carlo techniques for representing precisely the scattering events as well as towards the improvement of the shape of the beads. 
The number of generated spheres is about 29,000 and use of Monte Carlo techniques may not be the best choice to simulate the X-ray transport. The available Monte Carlo simulator can simulate only serial events [37]. In order to use general purpose shower packages with parallel processing facilities, the phantom should be converted to their format. Reported in literature simulations concerning computer-based phantoms are composed of a much smaller number of objects. This issue will be a challenging task and is already planned.

Once validated, this software tool may be used to design and evaluate complex imaging chains. Testing and evaluating different scenarios for this imaging technique saves time and is cost effective, especially before real practical work. In-silico results may be useful to optimize planned experimental work and imaging setups prior to the practical work.

\section{Conclusions}

This paper presented the evaluation of a computational breast phantom, which closely mimics the structure (shape, dimensions and content) and X-ray imaging characteristics of the corresponding real physical breast phantom. The subjective and quantitative comparison showed a very good agreement between real and simulated planar and tomographic images from the phantoms. Moreover, the additional virtual experiments with beads simulated from adipose (phantom 3) and polyethylene (phantom 4) showed that quantitatively and visually the images from these phantoms are close to those, generated from phantom 2 - where the filling material was water. The computed values for the $\beta$ parameter and the fractal dimension in case of 3D imaging are higher for the real images compared to the simulated ones indeed but the characteristics of the sharpness and scatter were not simulated. This is not a straightforward task though and has been planned as part of our future work. The application of the software platform is foreseen for the development and the evaluation of more realistic physical breast phantom dedicated to advanced breast imaging techniques.

\section{Acknowledgments}

This research work has been supported by MaXIMA project. This project has received funding from the European Union's Horizon 2020 research and innovation programme under grant agreement No 692097.

\section{References}

[1] Daniaux M, De Zordo T, Santner W, Amort B, Koppelstätter F, Jaschke W, et al. Dual-energy contrast-enhanced spectral mammography (CESM). Arch Gynecol Obstet 2015;292:739-47.

[2] Badr S, Laurent N, Régis C, Boulanger L, Lemaille S, Poncelet E. Dual-energy contrast-enhanced digital mammography in routine clinical practice in 2013. Diagn Interv Imaging 2014;95:245-58.

[3] Zhou SA, Brahme A. Development of phase-contrast X-ray imaging techniques and potential medical applications. Phys Medica 2008;24:129-48.

[4] Taibi A. Generalized subtraction methods in digital mammography. Eur ] Radiol 2009;72:447-53.

[5] Mettivier G, Fedon C, Di Lillo F, Longo R, Sarno A, Tromba G, et al. Glandular dose in breast computed tomography with synchrotron radiation. Phys Med Biol 2015;61:569-87.

[6] Sarno A, Mettivier G, Di Lillo F, Cesarelli M, Bifulco P, Russo P. Cone-beam micro computed tomography dedicated to the breast. Med Eng Phys 2016:38:1449-57.

[7] Sarno A, Mettivier G, Golosio B, Oliva P, Spandre G, Di Lillo F, et al. Imaging performance of phase-contrast breast computed tomography with synchrotron radiation and a CdTe photon-counting detector. Phys Medica 2016;32:681-90.

[8] Svahn TM, Houssami N, Sechopoulos I, Mattsson S. Review of radiation dose estimates in digital breast tomosynthesis relative to those in two-view fullfield digital mammography. Breast 2015;24:93-9.

[9] Sechopoulos I. A review of breast tomosynthesis. Part I. The image acquisition process. Med Phys 2013;40:014301.
[10] Sechopoulos I. A review of breast tomosynthesis. Part II. Image reconstruction, processing and analysis, and advanced applications. Med Phys 2013;40:014302.

[11] Sarno A, Mettivier G, Di Lillo F, Cesarelli M, Bifulco P, Russo P. Cone-beam micro computed tomography dedicated to the breast. Med Eng Phys 2016;38 (12):1449-57.

[12] Sarno A, Mettivier G, Russo P. Dedicated breast computed tomography: basic aspects. Med Phys 2015;42(6):2786-804.

[13] Kalender WA, Kolditz D, Steiding C, Ruth V, Lück F, Rößler A-C, Wenkel E. Technical feasibility proof for high-resolution low-dose photon-counting CT of the breast. Eur Radiol 2016:1-6.

[14] Bakic PR, Albert M, Brzakovic D, Maidment ADA. Mammogram synthesis using a 3D simulation. I. Breast tissue model and image acquisition simulation. Med Phys 2001;29(9):2131-9.

[15] Bakic PR, Albert M, Brzakovic D, Maidment ADA. Mammogram synthesis using a 3D simulation. II. Evaluation of synthetic mammogram texture. Med Phys 2002;29(9):2140-51.

[16] Bakic PR, Albert M, Brzakovic D, Maidment ADA. Mammogram synthesis using a three-dimensional simulation. III. Modeling and evaluation of the breast ductal network. Med Phys 2003;30(7):1914-25.

[17] Bliznakova K, Bliznakov Z, Bravou V, Kolitsi Z, Pallikarakis N. A threedimensional breast software phantom for mammography simulation. Phys Med Biol 2003;48(22):3699-719.

[18] Bliznakova K, Kazakli S, Pallikarakis N. An optimised 3D breast phantom for Xray breast imaging techniques. Proc IFMBE 2008;22(3):2455-8.

[19] Li CM, Segars WP, Tourassi GD, Boone JM, Dobbins JT. Methodology for generating a 3D computerized breast phantom from empirical data. Med Phys 2009;36(7):3122-31.

[20] Lau BA, Reiser I, Nishikawa RM, Bakic PR. A statistically defined anthropomorphic software breast phantom. Med Phys 2012;39(6):3375-85.

[21] Carton A-K, Bakic P, Ullberg C, Derand H, Maidment ADA. Development of a physical 3D anthropomorphic breast phantom. Med Phys 2011;38(2): 891-6.

[22] Park S, Jennings R, Liu H, Badano A, Myers K. A statistical, task-based evaluation method for three-dimensional x-ray breast imaging systems using variable-background phantoms. Med Phys 2010;37(12):6253-70.

[23] Chen B et al. An anthropomorphic breast model for breast imaging simulation and optimization. Acad Radiol 2011;18(5):536-46.

[24] Pokrajac DD, Maidment ADA, Bakic PR. Optimized generation of high resolution breast anthropomorphic software phantoms. Med Phys 2012;39 (4):2290-302.

[25] Zhang C, Bakic PR, Maidment ADA. Development of an anthropomorphic breast software phantom based on region growing algorithm. Proc. SPIE 2008:6918. 69180V-1-69180V-10.

[26] Ma AKW, Gunn S, Darambara DG. Introducing DeBRa: a detailed breast model for radiological studies. Phys Med Biol 2009;54(14):4533-45.

[27] O'Connor JM, Das M, Didier C, Mah'd M, Glick SJ. Development of an ensemble of digital breast object models. Lect. Notes Comput. Sci. 2010;6136:54-61.

[28] Kiarashi $\mathrm{N}$ et al. Development of matched virtual and physical breast phantoms based on patient data. Proc. SPIE 2013;8668. 866805-1-866805-6.

[29] Cockmartin L, Marshall N, Bosmans H. Design and evaluation of a phantom with structured background for digital mammography and breast tomosynthesis, IWDM'12 Proceedings of the 11th international conference on Breast Imaging, Philadelphia, PA, 2012. p. 642-9.

[30] Cockmartin L, Bakic P, Bosmans H, Maidment ADA, Gall H, Zerhouni M, et al. Power spectrum analysis of an anthropomorphic breast phantom compared to patient data in 2D digital mammography and breast tomosynthesis. In: Fujita $\mathrm{H}$, Hara T, Muramatsu C, editors. Breast Imaging: 12th international workshop, IWDM 2014, June 29 - July 2, Gifu City, Japan: Springer International Publishing; 2014. p. 423-9.

[31] Cockmartin L, Bosmans H, Marshall NW. Comparative power law analysis of structured breast phantom and patient images in digital mammography and breast tomosynthesis. Med Phys 2013;40(8):081920.

[32] Cockmartin L, Marshall NW, Zhang G, Lemmens K, Shaheen E, Van Ongeval C, Fredenberg E, Dance DR, Salvagnini E, Michielsen K, Bosmans H. Design and application of a structured phantom for detection performance comparison between breast tomosynthesis and digital mammography. Phys Med Biol 2017;62:758-80

[33] Gang GJ, Tward DJ, Lee J, Siewerdsen JH. Anatomical background and generalized detectability in tomosynthesis and cone-beam CT. Med Phys 2010;37:1948-65.

[34] Bliznakova K. Development of breast software phantom dedicated for research and educational purposes. In: RAD2016 proceedings, 23-27 May 2016, University of Nis, Serbia, p. 1-6, ISBN: 978-86-6125-160-3.

[35] Bosmans H, Bliznakova K, Padovani R, Christofides S, Van Peteghem N, Tsapaki V, et al. An EC supported FP7 project for the training and education of medical physics experts in radiology. Radiat Protect Dosimet 2015;165(1-4): $518-2$.

[36] http://physics.nist.gov/cgi-bin/Star/compos.pl?ap.

[37] Bliznakova K, Speller R, Horrocks J, Liaparinos P, Kolitsi Z, Pallikarakis N, Experimental validation of a radiographic simulation code using breast phantom for X-ray imaging. Comput Biol Med 2010;40:208-14.

[38] Buliev I. Object-oriented approach in the programming of applications for the field of the computed tomography. Comput Sci Technol 2011;1:3-10. ISSN 1312-3335. 
[39] Feldkamp L, Davis L. Practical cone-beam algorithm. J Opt Soc Am 1984;1:612-9.

[40] Marinov S, Buliev I, Bliznakov Z. Software platform for evaluation of X-ray images: application for validation of anthropomorphic software breast model, Union of Scientists in Bulgaria - Plovdiv 139-43, 2016. ISSN 1311-9427.

[41] Byng JW, Boyd NF, Fishell E, Jong RA, Yaffe MJ. Automated analysis of mammographic densities. Phys Med Biol 1996;41:909-23.
[42] Bliznakova K, Suryanarayanan S, Karellas A, Pallikarakis N. Evaluation of an improved algorithm for producing realistic 3D breast software phantoms: Application for mammography. Med Phys 2010;37:5604-917.

[43] Bakic P, Carton A, Reiser I, Maidment A, Nishikawa R. In: Martí et al. (editor), An anthropomorphic software breast phantom for tomosynthesis simulation: power spectrum analysis of phantom projections, IWDM 2010, LNCS 6136, 2010. p. $452-8$. 